\title{
Design of an SH-SAW Sensor Array with High Sensitivity and Partial Selectivity Using Polymer-Plasticizer Coatings
}

\author{
Nicholas Post, Karthick Sothivelr, Florian Bender, Antonio J. Ricco*, Fabien Josse \\ Dept. of El. \& Comp. Engineering, Marquette University, Milwaukee, Wisconsin 53201, United States \\ ${ }^{*}$ Dept. of Electrical Engineering, Stanford University, Stanford, California 94305, United States \\ Fabien.Josse@marquette.edu
}

\begin{abstract}
:
Coatings based on polymer-plasticizer blends for SH-SAW (shear-horizontal surface acoustic wave) sensor arrays are investigated for direct liquid-phase measurement. Based on a combination of Hansen solubility parameter analysis and previous experiments with different blend mixing ratios, polymers and plasticizers were selected to provide high sensitivity to BTEX compounds (benzene, toluene, ethylbenzene, xylenes) for each sensor device while maximizing array chemical diversity. The sensitivity of each coating/analyte combination was studied for a range of polymer-plasticizer mixing ratios. To characterize the chemical diversity in a given sensor array, the ratios of sensitivities of the coatings and the response time constants for each coating/analyte combination were compared. Guidelines for the design of an effective sensor array are discussed.
\end{abstract}

Key words: Polymer-plasticizer coating, sensor array, BTEX, sensitivity, partial selectivity

\section{Introduction}

To assess the pervasive contamination of groundwater and surface water with petroleum products, sensor coatings with high sensitivity to dissolved BTEX compounds (benzene, toluene, ethylbenzene, xylene) are needed, especially in the presence of multiple interferents. The limited number of commercially available polymer materials with high sensitivity and partial selectivity to BTEX have motivated new approaches to sensor coating design. Previous results have shown promise in detection of BTEX compounds using specific polymer-plasticizer combinations [1], but systematic design guidelines for sensor arrays with high sensitivity and partial selectivity are still needed.

In this work, two strategies were employed to design a sensor array to detect BTEX compounds. The first created a sensor array composed entirely of polymerplasticizer blend coatings ("Array 1"), all of which showed high sensitivity to BTEX. Plasticizer type and mixing ratio were varied to provide chemical diversity in the array. The second strategy used coatings both with and without plasticizer ("Array 2") to increase chemical diversity while accepting slightly lower sensitivity for some coatings. In both approaches, coating sensitivities to interferents must be studied to develop practical sensor arrays.

\section{Results}

Polymers poly(isobutylene) (PIB) and poly(epichlorohydrin) (PECH) have previously been identified as suitable sensor coating materials [2] and therefore were used for Array 2. Plasticizers diisooctyl azelate (DIOA) and 1,2-cyclohexane dicarboxylic acid diisononyl ester (DINCH) blended with polystyrene
(PS) at specific ratios have shown promise as sensor coatings [1]; they were used for Array 1 . These plasticizers were selected after careful analysis of Hansen solubility parameters $\left(\delta_{\mathrm{d}}, \delta_{\mathrm{p}}\right.$, and $\delta_{\mathrm{h}}$, which represent intermolecular dispersion forces, polar forces, and hydrogen bonding forces, respectively) and relative energy differences (RED), which are derived from differences in Hansen parameters between the two components of a blend or mixture. For relevant compounds, these values can be found in Table 1 . RED $<1$ indicates good miscibility.

Table1: Differences between Hansen solubility parameters of $1^{\text {st }}$ and $2^{\text {nd }}$ compound $\left(\Delta \delta\right.$, in $\left.M P a^{0.5}\right)$, with quadratic sum $R_{a}$ and normalized RED values.

\begin{tabular}{|c|c|c|c|c|c|c|}
\hline $1^{\text {st }}$ & $2^{\text {nd }}$ & $\Delta \boldsymbol{\delta}_{\mathbf{d}}$ & $\Delta \boldsymbol{\delta}_{\mathbf{p}}$ & $\Delta \boldsymbol{\delta}_{\mathbf{h}}$ & $\mathbf{R}_{\mathbf{a}}$ & $\mathbf{R E D}$ \\
\hline DINCH & PS & -3.2 & 1.68 & 2.35 & 7.02 & 0.82 \\
\hline DIOA & PS & -2.4 & 0.2 & 5.5 & 7.30 & 0.85 \\
\hline Benzene & PS & -0.2 & -4.5 & -0.9 & 4.61 & 0.54 \\
\hline
\end{tabular}

The remaining two sensor coatings for each array are blends of PS with the plasticizer ditridecyl phthalate (DTP) which, though lacking Hansen solubility parameters, was selected for its chemical similarities to the plasticizer diisooctyl phthalate (DOP). The latter showed good compatibility with BTEX compounds but, due to its low molecular weight, had limited permanence in the blend in aqueous-phase measurements, i.e. it leached out of the coating. DTP has a significantly higher molecular weight than DOP, leading to increased permanence in the blend. Coating compositions are given as weight/weight ratios of plasticizer to total blend. Likewise, the composition of a coating solution is given as weight/weight ratio of plasticizer and polymer to that of the total solution. 
Blending ratios were optimized to maximize sensitivity to BTEX analytes while enabling low coating degradation and low leaching in aqueous measurements.

Sensor measurements were conducted in aqueous solutions of single BTEX compounds; their concentrations were independently measured using a gas chromatograph-photoionization detector system. Concentrations of 1,2,4-trimethylbenzene (TMB), a common interferent, were defined by careful dilution of stock solutions. Each response was corrected for linear baseline drift and subsequently modelled using an exponential fit to extract equilibrium frequency shift, $\Delta f$, and response time constant, $\tau$. Frequency shifts were normalized to 1 part per million (ppm) analyte concentration, and average sensitivity and time constant were calculated for each coating/analyte combination.

To visualize array chemical diversity, radial plots of $\tau$ and coating sensitivity ratios are shown in Fig. 1 (Array 1) and Fig. 2 (Array 2). Sensitivity is normalized with respect to $1 \mathrm{ppm}$ benzene, analyte molecular weight, and analyte solubility in water; normalized coating sensitivities for each of the two sensor arrays are compared in Fig. 3 (Array 1) and Fig. 4 (Array 2).

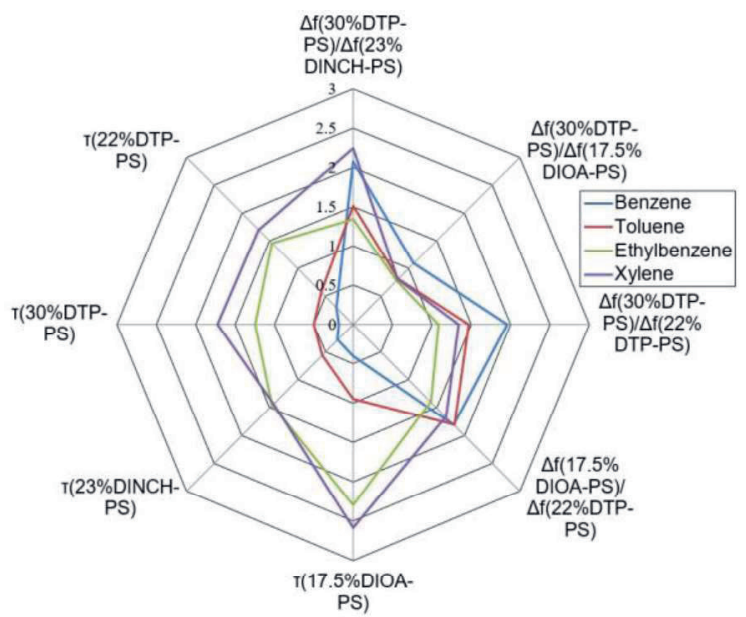

Fig. 1: Radial plot of polymer-plasticizer coated sensor array, displaying ratios of sensitivities of coatings and time constants (in units of $250 \mathrm{~s}$ ).

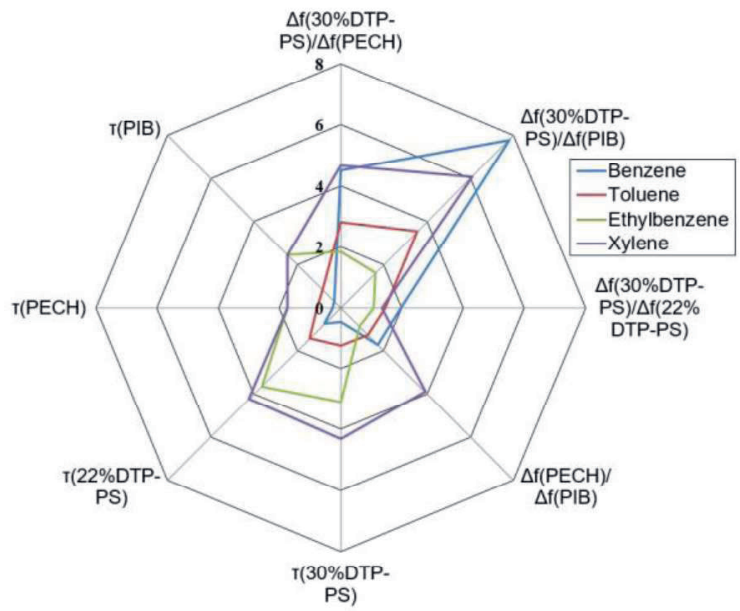

Fig. 2: Radial plot of polymer-plasticizer coated sensor array, displaying ratios of sensitivities of coatings $(\triangle f(30 \% D T P-P S) / \triangle f(P I B)$ scaled by $1 / 3)$ and time constants (in units of $100 \mathrm{~s}$ ).
As indicated by Figs. 1 and 3, a sensor array created entirely of polymer-plasticizer coatings provides good overall selectivity. Even higher selectivity is achieved by including commercially available polymers in the array, as indicated by Figs. 2 and 4, although this results in slightly lower sensitivity for some of the coatings.

Experiments using multiple DTP-PS sensor coatings with different mixing ratios suggest that it may be possible to create sensor arrays from a single polymerplasticizer pair, provided that the Hansen solubility parameters of the plasticizer are mismatched with respect to those of the polymer to provide sufficient chemical diversity in the array. Future work is planned to identify additional plasticizer candidates for such an array. While only TMB is tested here as an interferent, characterization of the responses of coated sensors to other common interferents, such as ethanol, naphthalene, and methyl tert-butyl ether (MTBE) will continue.

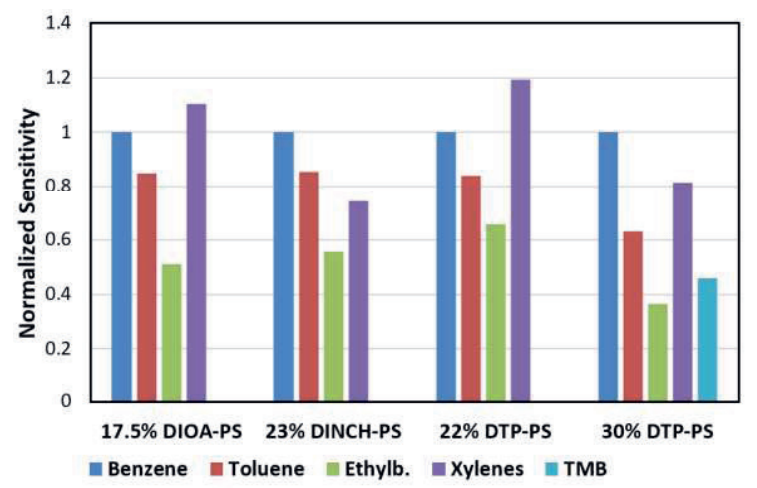

Fig. 3: Bar chart showing normalized sensitivity of the specified sensor coatings for Array 1.

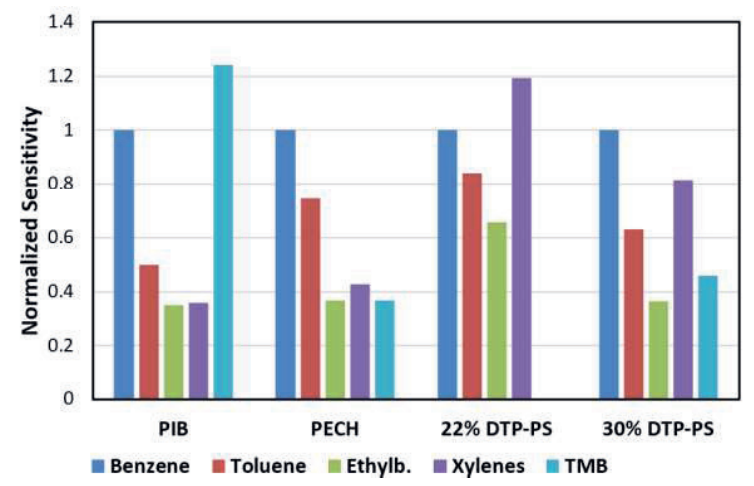

Fig. 4: Bar chart showing normalized sensitivity of the specified sensor coatings for Array 2.

\section{References:}

[1] P. Adhikari, L. Alderson, F. Bender, A.J. Ricco, and F. Josse, "Investigation of Polymer-Plasticizer Blends as SH-SAW Sensor Coatings for Detection of Benzene in Water with High Sensitivity and LongTerm Stability", ACS Sens., Vol. 2, pp. 157-164, 2017.

[2] F. Bender, R.E. Mohler, A.J. Ricco, and F. Josse, "Identification and Quantification of Aqueous Aromatic Hydrocarbons Using SH-Surface Acoustic Wave Sensors", Anal. Chem., Vol. 86, pp. 17941799, 2014. 